\title{
30 years of Inflammopharmacology
}

\author{
K. D. Rainsford ${ }^{1}$
}

Received: 25 January 2021 / Accepted: 27 January 2021 / Published online: 16 February 2021

(c) The Author(s), under exclusive licence to Springer Nature Switzerland AG part of Springer Nature 2021

It is now 30 years since the publication of the first issue in volume 1 of Inflammopharmacology (IPHM). The origins of this multi-disciplinary journal devoted to the science and clinical actions of drugs used in inflammatory diseases has been told previously (Rainsford 2016). It is fair to say that from humble beginnings IPHM has now become a mainstream journal for communicating research in the broad field of the pharmacology and therapy of inflammatory diseases and associated painful conditions. The science and clinical applications of inflammation pharmacology has advanced considerably since these earlier times. With these advances IPHM has also progressed. The Impact factor over 5 years has been 3.354 while that for 2019 (the most recent) was 3.238. The number of downloads to 2020 was 147,174 .

These and other metrics show the journal has wide and extensive interest in the multi-disciplinary field of the pharmaco-therapeutics of inflammatory diseases, be they from an infection, immunologic or other painful states. Papers that are submitted and published originate from most regions throughout the world.

Of full-text article requests made over recent publication years, among the main interest has been on NSAIDs and non-prescription (OTC) analgesics, use of probiotics in inflammatory bowel diseases, actions of zinc (alone or complexed), and Nigella sativa, curcuminoids, ivy leaf and other plant extracts and corticosteroids, as well as issues concerning the use of animal models in rheumatic disease research, and categorical assessments of DMARDs and NSAIDs in rheumatological diseases. Recent years have seen increasing interest in the effects of various chemically defined plant extracts and components on induced inflammatory reactions.

We have attempted to promote increasing high standards of manuscripts in the complex area of natural products by

K. D. Rainsford

editor@inflammopharmacology.com

1 Editor-in-Chief, Inflammopharmacology, Emeritus Professor of Biomedical Sciences, Biomedical Research Centre, Sheffield Hallam University, Howard Street, Sheffield S1 1WB, England, UK adherence to strict requirements as specified (Rainsford 2007). Among the recommendations are that manuscripts should present (a) a clear case with a testable hypothesis for studying the properties of the natural product, (b) the product should be chemical defined with quantitative details of the composition, (c) full dose-response data should be provided from at least 3 doses from all in vivo investigations, and at least 3 concentrations to enable concentration-response data for in vitro studies, (d) pharmacological data should also include experiments on the principally active components (preferably $\geq 25 \%$ of the material under investigation), (e) comprehensive analysis of the mechanisms of action(s) of the major components in relation to modern drug-receptor theories, and (f) all experiments should include a positive control or known standard for comparison.

Ethical justification should also be presented for the studies of inflammation and painful conditions in laboratory animal models. Though there is much interest in this area of natural products pharmacology, there is a need to establish the relevance of these investigations for the treatment of human conditions. Thus, in keeping with the journal remit to advance knowledge of the therapeutics of inflammatory diseases, there will be an increased focus on clinical relevance and human comparative studies on isolated components derived from defined natural products (see accompanying Editorial).

This issue has some articles on the life-long contributions of some leading scientists who have contributed to the development of inflammation pharmacology. The background and personal aspects concerning research in the field by these scientists have been a recurring theme in the journal. These articles show how the science has evolved from the longterm personal involvement of those who have shaped this broad field.

The success of IPHM over the years would not have been possible without the active participation and enthusiastic involvement of the editors and reviewers, for their help and guidance I am immensely grateful. It is worth noting that these people give their time and advice without recompense and reflecting their sincere academic and professional 
involvement in running and operation of the journal literally "for the good of all". Our collective views and application of the highest standards for published research is paramount.

Finally, I would like to express my sincere appreciation for all the invaluable help and guidance over the years from the Senior Editor in charge of IPHM, Dr Hans-Detlef Klüber, (Springer Nature Switzerland AG, 6330 Cham, Switzerland).

I hope we may continue to publish high-quality papers on leading research of high interest to the academic, professional and pharmaceutical communities. May this enthusiasm and support continue.

\section{References}

Rainsford KD (2007) Editorial: requirements for manuscripts on the pharmacological or inflammatory properties of natural products. Inflammapharmacology 15:179-180

Rainsford KD (2016) Origins of Inflammopharmacology. Twenty-five years on. Inflammopharmacology 24:297-302

Publisher's Note Springer Nature remains neutral with regard to jurisdictional claims in published maps and institutional affiliations. 